\title{
GLOBAL WELL-POSEDNESS FOR THE 2D MICROPOLAR RAYLEIGH-BÉNARD CONVECTION PROBLEM WITHOUT VELOCITY DISSIPATION
}

\author{
SHENG WANG
}

\begin{abstract}
In this article, we study the Cauchy problem to the micropolar Rayleigh-Bénard convection problem without velocity dissipation in two dimension. We first prove the local well-posedness of a smooth solution, and then establish a blow up criterion in terms of the gradient of scalar temperature field. At last, we obtain the global well-posedness to the system.
\end{abstract}

Key Words:2D micropolar Rayleigh-Bénard convection problem; blow-up criterion; smooth solution; global well-posedness.

\section{INTRODUCTION}

In fluid dynamics, the micropolar fluid model can be regarded as an extension of the classical fluid dynamics model represented by the Navier-Stokes equation, because the classical fluid dynamics model can't describe the microstructure. In 1965, C.A. Eringen first introduced equations to simulate micropolar fluids (see [8]). Interestingly, many experiments have shown that this model is more accurate than classical fluid dynamics models in describing some fluid movements, such as blood (see [12]). When we investigate the behavior of a fluid layer filling the area between two rigid surfaces, the fluid is heated from below. We usually describe this heat convection phenomenon in the framework of the Boussinesq equation approximation, which is called micropolar Rayleigh-Bénard convection problem(see [9,11,13]) and described by the following equations in $\mathbb{R}^{3}$

$$
\left\{\begin{array}{l}
u_{t}+u \cdot \nabla u-(\nu+\kappa) \Delta u+\nabla P=2 \kappa \nabla \times \omega+e_{3} \cdot \theta \\
\omega_{t}+u \cdot \nabla \omega-\gamma \Delta \omega-(\alpha+\beta) \nabla \operatorname{div} \omega+4 \kappa \omega=2 \kappa \nabla \times u \\
\theta_{t}+u \cdot \nabla \theta-\mu \Delta \theta=u \cdot e_{3} \\
\operatorname{div} u=0 \\
(u, \omega, \theta)(x, t)_{t=0}=\left(u_{0}, \omega_{0}, \theta_{0}\right)(x) \quad x \in \mathbb{R}^{3} .
\end{array}\right.
$$

where $u$ is the fluid velocity, $\omega$ is the field of microrotation representing the angular velocity of the rotation of the particles of the fluid, $P$ is the scalar pressure of the flow, $\theta$ is the scalar temperature, $\nu$ is the Newtonian kinematic viscosity, $\kappa$ is the micro-rotation viscosity, $\alpha$ and $\beta$ are the angular viscosities, $\mu$ is the thermal diffusivity, $e_{3}=(0,0,1)$ is the vertical unit vector, the forcing term $e_{3} \cdot \theta$ in the momentum equation describes the action of the buoyancy force on fluid motion, and $u \cdot e_{3}$ models the Rayleigh-Bénard convection in a heated fluid. Many recent efforts are focused on the 2D micropolar fluid with partial dissipation [6, 7, 14].

In present article, we shall consider the $2 \mathrm{D}$ micropolar Rayleigh-Bénard convection problem. For convenience, we assume that the velocity component in the 
$x_{3}$-direction is zero and the axes of rotation of particles are parallel to the $x_{3}$-axis. That is,

$$
u=u\left(x_{1}, x_{2}, 0\right), \quad \omega=\omega\left(0,0, \omega_{3}\left(x_{1}, x_{2}\right)\right), \quad P=P\left(x_{1}, x_{2}\right), \quad \theta=\theta\left(x_{1}, x_{2}\right),
$$

which implies that

$$
\operatorname{div} \omega=0, \quad \nabla \times u=\partial_{1} u_{2}-\partial_{2} u_{1}, \quad \nabla \times \omega=\left(\partial_{2} \omega,-\partial_{1} \omega\right), \quad \nabla \times \nabla \times \omega=-\Delta \omega .
$$

Thus, the 2D micropolar Rayleigh-Bénard convection problem can be written as

$$
\left\{\begin{array}{l}
u_{t}+u \cdot \nabla u-(\nu+\kappa) \Delta u+\nabla P=2 \kappa \nabla \times \omega+e_{2} \cdot \theta \\
\omega_{t}+u \cdot \nabla \omega-\gamma \Delta \omega+4 \kappa \omega=2 \kappa \nabla \times u \\
\theta_{t}+u \cdot \nabla \theta-\mu \Delta \theta=u \cdot e_{2} \\
\operatorname{div} u=0 \\
(u, \omega, \theta)(x, t)_{t=0}=\left(u_{0}, \omega_{0}, \theta_{0}\right)(x) .
\end{array}\right.
$$

In 2020, Xu et al. [15] first obtained global regularity for the system (1.1) with zero diffusivity (i.e., $\mu=0$ ). In 2021, Xu et al. 16 proved the unique local solvability of smooth solution when the system (1.1) has only velocity dissipation, and then established a criterion for the breakdown of smooth solutions imposed only the maximum norm of the gradient of scalar temperature field. Furthermore, they finally showed the global regularity of the system with zero angular viscosity (i.e., $\gamma=0)$.

However, the related results is still unknown for the case $\nu+\kappa=0$. The purpose of this work are to study the well-posedness of the following system (1.1) without velocity dissipation.

$$
\left\{\begin{array}{l}
u_{t}+u \cdot \nabla u+\nabla P=2 \kappa \nabla \times \omega+e_{2} \cdot \theta, \\
\omega_{t}+u \cdot \nabla \omega-\gamma \Delta \omega+4 \kappa \omega=2 \kappa \nabla \times u, \\
\theta_{t}+u \cdot \nabla \theta-\mu \Delta \theta=u \cdot e_{2}, \\
\operatorname{div} u=0, \\
(u, \omega, \theta)(x, t)_{t=0}=\left(u_{0}, \omega_{0}, \theta_{0}\right)(x) .
\end{array}\right.
$$

We first prove the local well-posedness of the smooth solution for the system (1.2), and then establish a blow up criterion in terms of the gradient of scalar temperature field and finally obtain global well-posedness of the system.

The main results in this paper are stated as follows.

Theorem 1.1. (Local well-posedness) Let $\gamma>0, \mu>0$, and $\operatorname{div} u_{0}=0$. Suppose $s>2$, and $\left(u_{0}, \omega_{0}, \theta_{0},\right) \in H^{s}\left(\mathbb{R}^{2}\right)$. Then there exist $T>0$ and a unique solution $(u, \omega, \theta)$ of the system (1.2) such that

$$
u \in C\left([0, T] ; H^{s}\left(\mathbb{R}^{2}\right)\right), \quad(\omega, \theta) \in C\left([0, T] ; H^{s}\left(\mathbb{R}^{2}\right)\right) \cap L^{2}\left(0, T ; H^{s+1}\left(\mathbb{R}^{2}\right)\right) .
$$

Theorem 1.2. (Blow-up criterion) Let $\gamma>0, \mu>0$, and $\operatorname{div} u_{0}=0$. Suppose $s>2,\left(u_{0}, \omega_{0}, \theta_{0},\right) \in H^{s}\left(\mathbb{R}^{2}\right)$ and solution $(u, \omega, \theta)$ of the system (1.2) in $H^{s}\left(\mathbb{R}^{2}\right)$ satisfies

$$
\|\nabla \theta(., \tau)\|_{L^{\infty}}<+\infty
$$

then $(u, \omega, \theta) \in H^{s}\left(\mathbb{R}^{2}\right)$ for all time $t \in[0 ; T]$. 
Theorem 1.3. (Global regularity) Let $\gamma>0, \mu>0$ and $\operatorname{div} u_{0}=0$. Suppose $s>2$, and $\left(u_{0}, \omega_{0}, \theta_{0},\right) \in H^{s}\left(\mathbb{R}^{2}\right)$. Then the system (1.2) has a unique global solution $(u, \omega, \theta)$ such that for any $T>0$,

$$
u \in C\left([0, \infty) ; H^{s}\left(\mathbb{R}^{2}\right)\right), \quad(\theta, \omega) \in C\left([0, \infty) ; H^{s}\left(\mathbb{R}^{2}\right)\right) \cap L^{2}\left(0, T ; H^{s+1}\left(\mathbb{R}^{2}\right)\right) .
$$

Here, let us explain some of the main difficulties and techniques involved in the process. First, the estimate of $\|\Omega\|_{L^{p}}:=\|\nabla \times u\|_{L^{p}}(2 \leq p \leq \infty)$ plays a very key role in our proof. In order to get it, the main difficulty is due to the dynamic microrotational term $\nabla \times \omega$ in the velocity equation. Indeed, taking a $\nabla \times$ on velocity equation of (1.2), we find that

$$
\Omega_{t}+u \cdot \nabla \Omega+2 \kappa \Delta \omega=\partial_{1} \theta .
$$

However, due to the non-dissipation property of the variable $u$, we have no obvious way to handle the term $\kappa \Delta \omega$ as a perturbation and force us to deal with $2 \kappa \Delta \omega$ as a whole. Here, to obtain the estimate of $\|\Omega\|_{L^{\infty}}$ so that we can get the estimate of $\|\nabla u\|_{L^{\infty}}$ and show the global regularity of the 2D micropolar fluid flows without velocity disspation, we used the idea in [7] and introduced a nice quantity $Z=\Omega+\frac{2 \kappa}{\gamma} \omega$ to overcome the difficulty. Fortunately, the key observation for the more complicated system (1.2) is still effective. Resort to the quantity $Z$, we can exploit the estimates of $\|\Omega\|_{L^{\infty}}$ and $\|\Omega\|_{L^{p}}$ and then establish a criterion for the breakdown of smooth solutions imposed only the maximum norm of the gradient of scalar temperature field by the classical logarithm inequality. In particular, this demonstrates that the extension of smooth solutions system (1.2) is dominated by the temperature field $\theta$ of the fluid. In the proof of the global regularity of the system without velocity dissipation, using the heat kernel estimation, we can obtain $\|\Omega\|_{L^{p}} \leq C\left(u_{0}, \omega_{0}, \theta_{0} ; T\right)(2 \leq p<\infty)$. Furthermore, combining with GagliardoNirenberg interpolation and the Calderon-Zygmund inequalities, one can obtain the key estimate of $\|u\|_{L^{\infty}}$. Based on it, making full use of the dissipation property of the variable $\theta$, we finally get the desired estimate $\|\nabla \theta\|_{L^{\infty}}$.

The rest of this article follows. First, we will recall some facts about the Littlewood-Paley decomposition, the frequency characterization of Sobolev space and Besov space, and the estimation of some nonlinear terms. Then, in Section 3, we present the proof of Theorem 1.1. Section 4 is devoted to the proof of Theorem 1.2 In the last section, we shall prove the global regularity of the system without velocity dissipation.

\section{Preliminaries and Lemmas}

In the preparatory section, we introduce some common notations, some basic points about Littlewood-Paley theory and compile some auxiliary lemmas.

Let $\mathcal{S}\left(\mathbb{R}^{2}\right)$ be the Schwartz class of rapidly decreasing function. Given $f \in \mathcal{S}\left(\mathbb{R}^{2}\right)$, its Fourier transform $\mathcal{F} f=\widehat{f}$ is defined by

$$
\widehat{f}(\xi)=\int_{\mathbb{R}^{2}} e^{-i x \cdot \xi} f(x) d x .
$$

Let $(\chi, \varphi)$ be a couple of smooth functions valued in $[0,1]$ such that $\chi$ is supported in the ball $\left\{\xi \in \mathbb{R}^{2}:|\xi| \leq \frac{4}{3}\right\}, \varphi$ is supported in the shell $\left\{\xi \in \mathbb{R}^{2}: \frac{3}{4} \leq|\xi| \leq \frac{8}{3}\right\}$, $\varphi(\xi)=\chi(\xi / 2)-\chi(\xi)$ and

$$
\chi(\xi)+\sum_{j \geq 0} \varphi\left(2^{-j} \xi\right)=1, \forall \xi \in \mathbb{R}^{2},
$$




$$
\sum_{j \in Z} \varphi\left(2^{-j} \xi\right)=1, \forall \xi \in \mathbb{R}^{2} \backslash\{0\} .
$$

First, we introduce the dyadic blocks $\Delta_{j}$ by setting

$\Delta_{j} f=0$ if $j \leq-2, \quad \Delta_{-1} f=\mathcal{F}^{-1}(\chi \mathcal{F} f)$ and $\quad \Delta_{j} f=\mathcal{F}^{-1}\left(\varphi\left(2^{-j} \cdot\right) \mathcal{F} f\right)$ if $j \geq 0$.

One may prove that for all tempered distribution $u$ the following Littlewood-Paley decomposition holds true:

$$
f=\sum_{j \geq-1} \Delta_{j} f, \quad S_{j} f=\sum_{-1 \leq q \leq j-1} \Delta_{q} f .
$$

One easily verifies that with our choice of $\varphi$,

$$
\Delta_{j} \Delta_{q} f \equiv 0, \quad \text { if } \quad|j-q| \geq 2 \quad \text { and } \quad \Delta_{j}\left(S_{q-1} f \Delta_{q} f\right) \equiv 0, \quad \text { if } \quad|j-q| \geq 5 .
$$

Let us recall the definition of the Besov space.

Definition 2.1. Let $s \in \mathbb{R}, 1 \leq p, r \leq+\infty$. The nonhomogeneous Besov space $B_{p, r}^{s}$ is defined by

$$
B_{p, r}^{s}=\left\{f \in \mathcal{S}^{\prime}\left(\mathbb{R}^{2}\right):\|f\|_{B_{p, r}^{s}}<+\infty\right\}
$$

where

$$
\|f\|_{B_{p, r}^{s}}:=\left\|2^{j s}\right\| \Delta_{j} f\left\|_{p}\right\|_{\ell^{r}} .
$$

Notice that the usual Sobolev space $H^{s}$ coincides with $B_{2,2}^{s}$ for every $s \in \mathbb{R}$, and the norm of $H^{s}$ can be characterized by

$$
\|f\|_{H^{s}} \approx\left\|S_{0} f\right\|_{L^{2}}+\left(\sum_{q \geq 0} 2^{2 q s}\left\|\Delta_{q} f\right\|_{L^{2}}^{2}\right)^{\frac{1}{2}} .
$$

We shall also use the following some important lemmas in the proof of our main results.

Lemma 2.1. We present some commutator estimates as follows (i) (Lemma 2.2 of [6] Let $s>0, f \in H^{s}\left(\mathbb{R}^{2}\right) \cap L^{\infty}\left(\mathbb{R}^{2}\right)$, and $g \in H^{s+1}\left(\mathbb{R}^{2}\right) \cap$ $W^{1, \infty}\left(\mathbb{R}^{2}\right)$ with $\operatorname{div} g=0$. Then the following calculus inequality holds

$$
\left\|\left[\Delta_{j}, g\right] \cdot \nabla f\right\|_{L^{2}} \leq C c_{j} 2^{-j s}\left(\|\nabla g\|_{L^{\infty}}\|f\|_{H^{s}}+\|\nabla g\|_{H^{s}}\|f\|_{L^{\infty}}\right),
$$

where $c_{j}$ is a sequence satisfying $\left\|c_{j}\right\|_{\ell^{2}} \leq 1$.

(ii) ((Lemma 2.100 of [3] ) Let $s>0, f \in H^{s}\left(\mathbb{R}^{2}\right) \cap W^{1, \infty}\left(\mathbb{R}^{2}\right)$, and $g \in H^{s}\left(\mathbb{R}^{2}\right) \cap$ $W^{1, \infty}\left(\mathbb{R}^{2}\right)$. Then the following calculus inequality holds

$$
\left\|\left[\Delta_{j}, g\right] \cdot \nabla f\right\|_{L^{2}} \leq C c_{j} 2^{-j s}\left(\|\nabla g\|_{L^{\infty}}\|f\|_{H^{s}}+\|g\|_{H^{s}}\|\nabla f\|_{L^{\infty}}\right),
$$

where $c_{j}$ is a sequence satisfying $\left\|c_{j}\right\|_{\ell^{2}} \leq 1$.

Lemma 2.2. ([5]) Let $j, k \in \mathbb{N}, 1 \leq a \leq b$ and $f \in L^{a}\left(\mathbb{R}^{2}\right)$. There exists a constant $C$ such that the following inequalities hold

$$
\begin{aligned}
\sup _{|\alpha|=k}\left\|\partial^{\alpha} S_{j} f\right\|_{L^{b}} & \leq C^{k} 2^{j\left(k+2\left(\frac{1}{a}-\frac{1}{b}\right)\right)}\left\|S_{j} f\right\|_{L^{a}}, \\
C^{-k} 2^{j k}\left\|\Delta_{j} f\right\|_{L^{a}} & \leq \sup _{|\alpha|=k}\left\|\partial^{\alpha} \Delta_{j} f\right\|_{L^{a}} \leq C^{k} 2^{j k}\left\|\Delta_{j} f\right\|_{L^{a}} .
\end{aligned}
$$


Lemma 2.3. ([10]) Let $s>0, f, g \in H^{s}\left(\mathbb{R}^{2}\right) \cap L^{\infty}\left(\mathbb{R}^{2}\right)$. Then the following inequality holds

$$
\|f g\|_{H^{s}} \leq C\left(\|f\|_{L^{\infty}}\|g\|_{H^{s}}+\|f\|_{H^{s}}\|g\|_{L^{\infty}}\right) .
$$

To prove this proposition, we recall the maximal regularity property for the heat kernel (see, [1]).

Lemma 2.4. The operator $T$ defined by

$$
T f(t)=\int_{0}^{t} \Delta e^{(t-s) \Delta} f(s) d s
$$

maps $L^{p}\left(0, T ; L^{q}\left(\mathbb{R}^{d}\right)\right)$ to $L^{p}\left(0, T ; L^{q}\left(\mathbb{R}^{d}\right)\right)$ for any $T \in(0, \infty)$ and $p, q \in(1, \infty)$.

\section{The Proof of Theorem 1.1}

This section is devoted to the proof of the local well-posedness for a smooth solution to the system (1.2). For a clear presentation, we split it into the following several steps.

Step 1. Construction of an approximate solution sequence.

Here we use the classical Friedrich's method: For $n \geq 1$, let $J_{n}$ be the spectral cut-off defined by

$$
\widehat{J_{n} f}(\xi)=1_{[0, n]}(|\xi|) \widehat{f}(\xi), \quad \xi \in \mathbb{R}^{2} .
$$

We consider the following system in the space $L_{n}^{2}:=\left\{f \in L^{2}\left(\mathbb{R}^{2}\right) \mid \operatorname{supp} f \subset\right.$ $B(0, n)\}$ :

$$
\left\{\begin{array}{l}
\partial_{t} u_{n}+\mathcal{P} J_{n}\left(J_{n} u_{n} \cdot \nabla J_{n} u_{n}\right)=2 \kappa \mathcal{P} \nabla \times \omega_{n}+\mathcal{P} J_{n}\left(\theta_{n} \cdot e_{2}\right), \\
\partial_{t} \omega_{n}+J_{n}\left(J_{n} u_{n} \cdot \nabla J_{n} \omega_{n}\right)-\gamma \Delta \omega_{n}+4 \kappa \omega_{n}=2 \kappa \mathcal{P} \nabla \times u_{n}, \\
\partial_{t} \theta_{n}+J_{n}\left(J_{n} u_{n} \cdot \nabla J_{n} \theta_{n}\right)-\mu \Delta \theta_{n}=\mathcal{P} J_{n}\left(u_{n} \cdot e_{2}\right), \\
\left.\left(u_{n}, \omega_{n}, \theta_{n}\right)\right|_{t=0}=J_{n}\left(u_{0}, \omega_{0}, \theta_{0}\right) .
\end{array}\right.
$$

Here $\mathcal{P}$ denotes the Helmholtz projection operator. The Cauchy-Lipschitz theorem entails that the system (3.1) exists a unique maximal solution $\left(u_{n}, \omega_{n}, \theta_{n}\right)$ in $\mathcal{C}^{1}\left(\left[0, T_{n}^{*}\right) ; L_{n}^{2}\right)$. On the other hand, we observe that $J_{n}^{2}=J_{n}, \mathcal{P}^{2}=\mathcal{P}$ and $J_{n} \mathcal{P}=\mathcal{P} J_{n}$. It follows that $\left(\mathcal{P} u_{n}, \omega_{n}, \theta_{n}\right)$ and $\left(J_{n} \mathcal{P} u_{n}, J_{n} \omega_{n}, J_{n} \theta_{n}\right)$ are also solutions to the system (3.1). The uniqueness gives that $J_{n} u_{n}=u_{n}, J_{n} \omega_{n}=\omega_{n}$ and $J_{n} \theta_{n}=\theta_{n}$. Therefore,

$$
\left\{\begin{array}{l}
\partial_{t} u_{n}+\mathcal{P} J_{n}\left(u_{n} \cdot \nabla u_{n}\right)=2 \kappa \mathcal{P} \nabla \times \omega_{n}+\mathcal{P} J_{n}\left(\theta_{n} \cdot e_{2}\right), \\
\partial_{t} \omega_{n}+J_{n}\left(u_{n} \cdot \nabla \omega_{n}\right)-\gamma \Delta \omega_{n}+4 \kappa \omega_{n}=2 \kappa \mathcal{P} \nabla \times u_{n}, \\
\partial_{t} \theta_{n}+J_{n}\left(u_{n} \cdot \nabla \theta_{n}\right)-\mu \Delta \theta_{n}=\mathcal{P} J_{n}\left(u_{n} \cdot e_{2}\right), \\
\left.\left(u_{n}, \omega_{n}, \theta_{n}\right)\right|_{t=0}=J_{n}\left(u_{0}, \omega_{0}, \theta_{0}\right) .
\end{array}\right.
$$

As the operators $J_{n}$ and $\mathcal{P} J_{n}$ are the orthogonal projectors for the $L^{2}$-inner product, the above formal calculations remain unchanged.

Step 2. Energy estimates.

$$
\left\|u_{n}\right\|_{L^{2}}^{2}+\left\|\omega_{n}\right\|_{L^{2}}^{2}+\left\|\theta_{n}\right\|_{L^{2}}^{2}+2 \gamma \int_{0}^{t}\left\|\nabla \omega_{n}(\tau)\right\|_{L^{2}}^{2} d \tau+2 \mu \int_{0}^{t}\left\|\nabla \theta_{n}(\tau)\right\|_{L^{2}}^{2} d \tau \leq C\left(u_{0}, \omega_{0}, \theta_{0} ; T\right) .
$$


Next we present the uniform estimates for the approximate solutions $\left(u_{n}, \omega_{n}, \theta_{n}\right)$ in $H^{s}$. Applying the operator $\Delta_{j}(j \geq 0)$ to the system (3.2), and taking the $L^{2}$ scalar product of the first equation of (3.2) with $\Delta_{j} u_{n}$, the second equation with $\Delta_{j} \omega_{n}$, and the third equation with $\Delta_{j} \theta_{n}$ respectively, by the condition $\operatorname{div} u_{n}=0$, we obtain

$$
\begin{aligned}
& \frac{1}{2} \frac{d}{d t}\left(\left\|\Delta_{j} u_{n}\right\|_{L^{2}}^{2}+\left\|\Delta_{j} \omega_{n}\right\|_{L^{2}}^{2}+\left\|\Delta_{j} \theta_{n}\right\|_{L^{2}}^{2}\right)+\gamma\left\|\nabla \Delta_{j} \omega_{n}\right\|_{L^{2}}^{2}+\mu\left\|\nabla \Delta_{j} \theta_{n}\right\|_{L^{2}}^{2} \\
& =-\left(\left[\Delta_{j}, u_{n}\right] \nabla u_{n}, \Delta_{j} u_{n}\right)_{L^{2}}-\left(\left[\Delta_{j}, u_{n}\right] \nabla \omega_{n}, \Delta_{j} \omega_{n}\right)_{L^{2}}-\left(\left[\Delta_{j}, u_{n}\right] \nabla \theta_{n}, \Delta_{j} \theta_{n}\right)_{L^{2}} \\
& \quad+\kappa\left(\Delta_{j} \nabla \times \omega_{n}, \Delta_{j} u_{n}\right)_{L^{2}}+\kappa\left(\Delta_{j} \nabla \times u_{n}, \Delta_{j} \omega_{n}\right)_{L^{2}} \\
& \quad+\left(\Delta_{j} \theta_{n}, \Delta_{j} u_{n}\right)_{L^{2}}+\left(\Delta_{j} u_{n}, \Delta_{j} \theta_{n}\right)_{L^{2}} .
\end{aligned}
$$

Employing Lemma 2.1 and the Young inequality, multiplying both sides by $2^{2 j s}$, summing over $j \geq 0$, and then combining with (3.3), we thus get

$$
\begin{aligned}
& \frac{1}{2} \frac{d}{d t}\left(\left\|u_{n}\right\|_{H^{s}}^{2}+\left\|\omega_{n}\right\|_{H^{s}}^{2}+\left\|\theta_{n}\right\|_{H^{s}}^{2}\right)+\gamma\left\|\nabla \omega_{n}\right\|_{H^{s}}^{2}+\mu\left\|\nabla \theta_{n}\right\|_{H^{s}}^{2} \\
& \leq C\left(1+\left\|\nabla u_{n}\right\|_{L^{\infty}}+\left\|\nabla \omega_{n}\right\|_{L^{\infty}}+\left\|\nabla \theta_{n}\right\|_{L^{\infty}}\right)\left(\left\|u_{n}\right\|_{H^{s}}^{2}+\left\|\omega_{n}\right\|_{H^{s}}^{2}+\left\|\theta_{n}\right\|_{H^{s}}^{2}\right),
\end{aligned}
$$

from which and the Gronwall inequality, it follows that

$$
E_{n}(t) \leq\left(\left\|u_{0}\right\|_{H^{s}}^{2}+\left\|\omega_{0}\right\|_{H^{s}}^{2}+\left\|\theta_{0}\right\|_{H^{s}}^{2}\right) \exp \left(\int_{0}^{t} G_{n}(\tau) d \tau\right),
$$

where

$$
\begin{aligned}
& E_{n}(t)=\left(\left\|u_{n}\right\|_{H^{s}}^{2}+\left\|\omega_{n}\right\|_{H^{s}}^{2}+\left\|\theta_{n}\right\|_{H^{s}}^{2}\right)+2 \gamma \int_{0}^{t}\left\|\nabla \omega_{n}(\tau)\right\|_{H^{s}}^{2} d \tau+2 \mu \int_{0}^{t}\left\|\nabla \theta_{n}(\tau)\right\|_{H^{s}}^{2} d \tau, \\
& G_{n}(t)=1+\left\|\nabla u_{n}(t)\right\|_{L^{\infty}}+\left\|\nabla \omega_{n}(t)\right\|_{L^{\infty}}+\left\|\nabla \theta_{n}(t)\right\|_{L^{\infty}} .
\end{aligned}
$$

Step 3. Uniform estimates and existence of the solution. We denote $T_{n}^{*}$ by the maximal existence time of the solution $\left(u_{n}, \omega_{n}, \theta_{n}\right)$ and define

$$
\tilde{T}_{n}^{*}=\sup \left\{t \in\left[0, T_{n}^{*}\right): E_{n}(\tau) \leq 2\left(\left\|u_{0}\right\|_{H^{s}}^{2}+\left\|\omega_{0}\right\|_{H^{s}}^{2}+\left\|\theta_{0}\right\|_{H^{s}}^{2}\right) \quad \text { for } \quad \tau \in[0, t]\right\} .
$$

From (3.5) and the Sobolev embedding $(s>2)$, we find that

$$
E_{n}(t) \leq\left(\left\|u_{0}\right\|_{H^{s}}^{2}+\left\|\omega_{0}\right\|_{H^{s}}^{2}+\left\|\theta_{0}\right\|_{H^{s}}^{2}\right) \exp \left(C\left(1+\left\|u_{0}\right\|_{H^{s}}^{2}+\left\|\omega_{0}\right\|_{H^{s}}^{2}+\left\|\theta_{0}\right\|_{H^{s}}^{2}\right) t\right), t \in\left[0, \tilde{T}_{n}^{*}\right) .
$$

Taking $T$ small enough such that

$$
\exp \left(C\left(1+\left\|u_{0}\right\|_{H^{s}}^{2}+\left\|\omega_{0}\right\|_{H^{s}}^{2}+\left\|\theta_{0}\right\|_{H^{s}}^{2}\right) T\right) \leq \frac{3}{2} .
$$

Now we can conclude that $\tilde{T}_{n}^{*} \geq T$. Otherwise, we have

$$
E_{n}(t) \leq \frac{3}{2}\left(\left\|u_{0}\right\|_{H^{s}}^{2}+\left\|\omega_{0}\right\|_{H^{s}}^{2}+\left\|\theta_{0}\right\|_{H^{s}}^{2}\right) \quad \text { for } \quad t \in\left[0, \tilde{T}_{n}^{*}\right],
$$

which contradicts with the definition of $\tilde{T}_{n}^{*}$. Thus, the approximate solution $\left(u_{n}, \omega_{n}, \theta_{n}\right)$ exists on $[0, T]$ and satisfies the following uniform estimates

$$
E_{n}(t) \leq 2\left(\left\|u_{0}\right\|_{H^{s}}^{2}+\left\|\omega_{0}\right\|_{H^{s}}^{2}+\left\|\theta_{0}\right\|_{H^{s}}^{2}\right) \quad \text { for } \quad t \in[0, T] .
$$

On the other hand, it is easy to verify by using equations (3.2), uniform estimates (3.6) and Lemma2.3 that $\left(\partial_{t} u_{n}, \partial_{t} \omega_{n}, \partial_{t} \theta_{n}\right)$ is uniformly bounded in $L^{2}\left(0, T ; H^{s-1}\left(\mathbb{R}^{2}\right)\right)$. Thus, the Aubin-Lions compactness theorem (see e.g. [2]) ensures that there exists 
a subsequence $\left(u_{n_{k}}, \omega_{n_{k}}, \theta_{n_{k}}\right)$ of $\left(u_{n}, \omega_{n}, \theta_{n}\right)$ converging to a family function $(u, \omega, \theta)$ such that

$$
u \in L^{\infty}\left(0, T ; H^{s}\left(\mathbb{R}^{2}\right)\right),(\omega, \theta) \in L^{\infty}\left(0, T ; H^{s}\left(\mathbb{R}^{2}\right)\right) \cap L^{2}\left(0, T ; H^{s+1}\left(\mathbb{R}^{2}\right)\right) .
$$

Then passing to limit in (3.2), it is easy to see that $(u, \omega, \theta)$ satisfies the system (1.2).

Step 4. Continuity in time of the solution.

Revisiting the proof of Step 2, one can in fact obtain better uniform estimates for $\left(u_{n}, \omega_{n}, \theta_{n}\right)$ (thus for $\left.(u, \omega, \theta)\right)$ :

$$
\|u\|_{\tilde{L}^{\infty}\left(0, T ; H^{s}\left(\mathbb{R}^{2}\right)\right)}+\|\omega\|_{\tilde{L}^{\infty}\left(0, T ; H^{s}\left(\mathbb{R}^{2}\right)\right)}+\|\theta\|_{\tilde{L}^{\infty}\left(0, T ; H^{s}\left(\mathbb{R}^{2}\right)\right)} \leq C,
$$

where $\|f\|_{\tilde{L}^{\infty}\left(0, T ; H^{s}\left(\mathbb{R}^{2}\right)\right)}=\sum_{j \geq-1} 2^{2 j s}\left\|\Delta_{j} f\right\|_{L^{\infty}\left(0, T ; L^{2}\right)}^{2}$. Then we can conclude $(u, \omega, \theta) \in C\left([0, T] ; H^{s}\left(\mathbb{R}^{2}\right)\right)$. Indeed, for any $\varepsilon>0$, we can take $N$ big enough such that

$$
\sum_{j>N} 2^{2 j s}\left\|\Delta_{j} u\right\|_{L^{\infty}\left(0, T ; L^{2}\right)}^{2} \leq \frac{\varepsilon}{4}
$$

For any $t \in(0, T)$ and $\sigma$ such that $t+\sigma \in[0, T]$, we have

$$
\begin{aligned}
\|u(t+\sigma)-u(t)\|_{H^{s}}^{2} & \leq \sum_{-1 \leq j \leq N} 2^{2 j s}\left\|\Delta_{j}(u(t+\sigma)-u(t))\right\|_{L^{2}}^{2}+\frac{\varepsilon}{2} \\
& \leq \sum_{-1 \leq j \leq N} 2^{2 j s}|\sigma|\left\|\partial_{t} u\right\|_{L^{2}\left(0, T ; L^{2}\right)}^{2}+\frac{\varepsilon}{2} \\
& \leq 2 N 2^{2 N s}|\sigma|\left\|\partial_{t} u\right\|_{L^{2}\left(0, T ; L^{2}\right)}^{2}+\frac{\varepsilon}{2} \leq \varepsilon,
\end{aligned}
$$

for $|\sigma|$ small enough. Hence, $u(t)$ is continuous in $H^{s}\left(\mathbb{R}^{2}\right)$ at the time $t$. Similarly, we can obtain $(\omega, \theta) \in C\left([0, T] ; H^{s}\left(\mathbb{R}^{2}\right)\right)$.

Step 5. Uniqueness of the solution.

Let $\left(u_{1}, \omega_{1}, \theta_{1}\right)$ and $\left(u_{2}, \omega_{2}, \theta_{2}\right)$ be two solutions of (1.2) with the same initial data. We denote $\delta u=u_{1}-u_{2}, \delta \omega=\omega_{1}-\omega_{2}, \delta \theta=\theta_{1}-\theta_{2}$ and $\delta P=0$. Then $(\delta u, \delta \omega, \delta \theta)$ satisfies

$$
\left\{\begin{array}{l}
\partial_{t} \delta u+u_{2} \cdot \nabla \delta u=-\delta u \cdot \nabla u_{1}+2 \kappa \nabla \times \delta \omega+\delta \theta e_{2} \\
\partial_{t} \delta \omega+u_{2} \cdot \nabla \delta \omega-\gamma \Delta \delta \omega+4 \kappa \delta \omega=-\delta u \cdot \nabla \omega_{1}+2 \kappa \nabla \times \delta u \\
\partial_{t} \delta \theta+u_{2} \cdot \nabla \delta \theta-\mu \Delta \delta \theta=-\delta u \cdot \nabla \theta_{1}+\delta u e_{2}, \\
\left.(\delta u, \delta \theta, \delta \phi)\right|_{t=0}=(0,0,0) .
\end{array}\right.
$$

Taking $L^{2}\left(\mathbb{R}^{2}\right)$ energy estimates, it is easy to show that

$$
\frac{d}{d t}\left(\|\delta u\|_{L^{2}}^{2}+\|\delta \omega\|_{L^{2}}^{2}+\|\delta \theta\|_{L^{2}}^{2}\right) \leq C\left(\|\delta u\|_{L^{2}}^{2}+\|\delta \omega\|_{L^{2}}^{2}+\|\delta \theta\|_{L^{2}}^{2}\right),
$$

which along with the Gronwall inequality implies $(\delta u, \delta \omega, \delta \theta)=(0,0,0)$. The proof of Theorem 1.1 is completed.

\section{The Proof of Theorem 1.2}

This section is devoted to the proof of blow-up criterion for smooth solutions to the system (1.2). First, for the basic $L^{2}$-estimate and the $\dot{H}^{1}$-estimate of the system (1.1), we readily get 


$$
\|u\|_{L^{2}}^{2}+\|\omega\|_{L^{2}}^{2}+\|\theta\|_{L^{2}}^{2}+\int_{0}^{t}\left(\|\nabla \omega\|_{L^{2}}^{2}+\|\nabla \theta\|_{L^{2}}^{2}\right) d \tau \leq C\left(u_{0}, \omega_{0}, \theta_{0} ; T\right)
$$

and

$$
\|\nabla u\|_{L^{2}}^{2}+\|\nabla \omega\|_{L^{2}}^{2}+\|\nabla \theta\|_{L^{2}}^{2}+\int_{0}^{t}\left(\left\|\nabla^{2} \omega\right\|_{L^{2}}^{2}+\left\|\nabla^{2} \theta\right\|_{L^{2}}^{2}\right) d \tau \leq C\left(u_{0}, \omega_{0}, \theta_{0} ; T\right) .
$$

Denote the vorticity of velocity $u$ by $\Omega=\nabla \times u$. Applying the operator $\nabla \times$ to the first equation of (1.2) yield that

$$
\Omega_{t}+u \cdot \nabla \Omega+2 \kappa \Delta \omega=\partial_{1} \theta .
$$

Due to the lack of volecity viscosity, we have no obvious way to handle $\Delta \omega$. As in [7, set

$$
Z=\Omega+\frac{2 \kappa}{\gamma} \omega
$$

Thus, we can take advantage of its evolution in time governed by

$$
Z_{t}+u \cdot \nabla Z=\frac{4 \kappa^{2}}{\gamma} Z-\frac{16 \kappa^{3}}{\gamma} \omega+\partial_{1} \theta .
$$

This leads to the following $L^{p}$-estimate of $Z$ after multiplying (4.4) by $|Z|^{p-2} Z$ with $p \geq 2$, integrating over $\mathbb{R}^{2}$, using the incompressibility of $u$ and the Hölder inequality, we have

$$
\frac{1}{p} \frac{d}{d t}\|Z\|_{L^{p}}^{p} \leq \frac{16 \kappa^{3}}{\gamma}\|\omega\|_{L^{p}}\|Z\|_{L^{p}}^{p-1}+\frac{4 \kappa^{2}}{\gamma}\|Z\|_{L^{p}}^{p}+\|\nabla \theta\|_{L^{p}}\|Z\|_{L^{p}}^{p-1}
$$

Dividing by $\|Z\|_{L^{p}}^{p-1}$ and integrating in time yield

$$
\|Z\|_{L^{p}} \leq\left\|Z_{0}\right\|_{L^{p}}+C \int_{0}^{t}\left(\|\omega(., \tau)\|_{L^{p}}+\|\nabla \theta(., \tau)\|_{L^{p}}\right) d \tau .
$$

In particular,

$$
\|Z\|_{L^{\infty}} \leq\left\|Z_{0}\right\|_{L^{\infty}}+C \int_{0}^{t}\left(\|\omega(., \tau)\|_{L^{\infty}}+\|\nabla \theta(., \tau)\|_{L^{\infty}}\right) d \tau .
$$

From the second equation of (1.2), we have

$$
\|\omega\|_{L^{p}} \leq\left\|\omega_{0}\right\|_{L^{p}}+C \int_{0}^{t}\|\nabla \times u(., \tau)\|_{L^{p}} d \tau,
$$

which together with (4.3) implies that

$$
\|\omega\|_{L^{p}} \leq\left\|\omega_{0}\right\|_{L^{p}}+C \int_{0}^{t}\left(\|\omega(., \tau)\|_{L^{p}}+\|Z(., \tau)\|_{L^{p}}\right) d \tau,
$$

and taking limit $p \rightarrow \infty$ lead to

$$
\|\omega\|_{L^{\infty}} \leq\left\|\omega_{0}\right\|_{L^{\infty}}+C \int_{0}^{t}\left(\|\omega(., \tau)\|_{L^{\infty}}+\|Z(., \tau)\|_{L^{\infty}}\right) d \tau .
$$


On the other hand, we need make $L^{p}$-estimate of $\nabla \theta$. Taking $\nabla^{\perp}=\left(-\partial_{x 2}, \partial_{x 1}\right)$ to the third equation of (1.2), we obtain

$$
\nabla^{\perp} \theta_{t}+u \cdot \nabla \nabla^{\perp} \theta-\mu \Delta \nabla^{\perp} \theta=\nabla^{\perp} \theta \cdot \nabla u+\nabla u .
$$

Taking $L^{2}$ inner product (4.9) with $\left|\nabla^{\perp} \theta\right|^{p-2} \nabla^{\perp} \theta(2 \leq p<\infty)$, using the incompressibility of $u$ and the Hölder inequality, we have

$$
\frac{1}{p} \frac{d}{d t}\|\nabla \theta\|_{L^{p}}^{p} \leq\|\nabla u\|_{L^{p}}\|\nabla \theta\|_{L^{\infty}}\|\nabla \theta\|_{L^{p}}^{p-1}+\|\nabla u\|_{L^{p}}\|\nabla \theta\|_{L^{p}}^{p-1} .
$$

Dividing by $\|\nabla \theta\|_{L^{p}}^{p-1}$ and integrating in time yield

$$
\begin{aligned}
\|\nabla \theta\|_{L^{p}} & \leq\left\|\nabla \theta_{0}\right\|_{L^{p}}+C \int_{0}^{t}\left(1+\|\nabla \theta(., \tau)\|_{L^{\infty}}\right)\|\nabla u\|_{L^{p}} d \tau \\
& \leq\left\|\nabla \theta_{0}\right\|_{L^{p}}+C \int_{0}^{t}\left(1+\|\nabla \theta(., \tau)\|_{L^{\infty}}\right)\|\nabla \times u\|_{L^{p}} d \tau \\
& \leq\left\|\nabla \theta_{0}\right\|_{L^{p}}+C \int_{0}^{t}\left(1+\|\nabla \theta(., \tau)\|_{L^{\infty}}\right)\left(\|Z\|_{L^{p}}+\|\omega\|_{L^{p}}\right) d \tau .
\end{aligned}
$$

Due to

$$
\|\nabla \theta(., \tau)\|_{L^{\infty}}<+\infty
$$

combining with (4.5), (4.7) and (4.10), and applying the Gronwall inequality, we have

$\|Z\|_{L^{p}}+\|\omega\|_{L^{p}}+\|\nabla \theta\|_{L^{p}} \leq\left(\left\|\Omega_{0}\right\|_{L^{p}}+\left\|\omega_{0}\right\|_{L^{p}}+\left\|\nabla \theta_{0}\right\|_{L^{p}}\right) \exp \left(C \int_{0}^{t}\left(1+\|\nabla \theta(., \tau)\|_{L^{\infty}}\right) d \tau\right)$,

for $2 \leq p<\infty$.

Furthermore, employing (4.6) and (4.8), and then applying the Gronwall inequality, we obtain

$$
\|Z\|_{L^{\infty}}+\|\omega\|_{L^{\infty}} \leq C\left(\left\|\Omega_{0}\right\|_{L^{\infty}}+\left\|\omega_{0}\right\|_{L^{\infty}}+\left\|\nabla \theta_{0}\right\|_{L^{p}}\right) e^{C t} \leq C\left(u_{0}, \omega_{0}, \theta_{0} ; T\right) .
$$

From (4.12) and (4.13), we finally conclude that

$$
\|\Omega\|_{L^{p}}+\|\Omega\|_{L^{\infty}}<+\infty .
$$

Now, recall the following well-known result (see e.g. 4]):

$$
\|\nabla u\|_{L^{\infty}} \leq C\left\{1+\|\Omega\|_{L^{p}}+\|\Omega\|_{L^{\infty}} \ln \left(1+\|u\|_{H^{s}}\right)\right\} .
$$

From (4.14), the above inequality implies that

$$
\|\nabla u\|_{L^{\infty}} \leq C \ln \left(1+\|u\|_{H^{s}}\right) .
$$

We write the equation of $\omega(t)$ in (1.2) as

$$
\omega(t)=e^{\gamma t \Delta} u_{0}+\int_{0}^{t} e^{\gamma(t-\tau) \Delta}(2 \kappa \nabla \times u-4 \kappa \omega-u \cdot \nabla \omega) d \tau .
$$

Taking $p=2$ and $2 \leq q<\infty$ in Lemma 2.5, we get

$$
\int_{0}^{t}\|\Delta \omega\|_{L^{q}}^{2} d \tau \leq C\left\|u_{0}\right\|_{H^{2}}^{2}+C \int_{0}^{t}\|\nabla \times u\|_{L^{q}}^{2}+\|\omega\|_{L^{q}}^{2}+\|u \cdot \nabla \omega\|_{L^{q}}^{2} d \tau .
$$


On the other hand, using the Sobolev embedding and combining with (4.1) and (4.2), we have

$$
\begin{aligned}
\int_{0}^{t}\|\omega\|_{L^{q}}^{2} d \tau \leq C & \int_{0}^{t}\|\nabla \omega\|_{L^{2}}^{2} d \tau \leq C\left(u_{0}, \omega_{0}, \theta_{0} ; T\right), \\
\int_{0}^{t}\|u \cdot \nabla \omega\|_{L^{q}}^{2} d \tau & \leq \int_{0}^{t}\|u\|_{L^{2 q}}^{2}\|\nabla \omega\|_{L^{2 q}}^{2} d \tau \\
& \leq C \int_{0}^{t}\|\nabla u\|_{L^{2}}^{2}\left\|\nabla^{2} \omega\right\|_{L^{2}}^{2} d \tau \\
& \leq C \sup _{t \in(0, T)}\|\nabla u\|_{L^{2}}^{2} \int_{0}^{t}\left\|\nabla^{2} \omega\right\|_{L^{2}}^{2} d \tau \\
& \leq C\left(u_{0}, \theta_{0}, \omega_{0} ; T\right) .
\end{aligned}
$$

Thus, from (4.17) we get

$$
\int_{0}^{t}\|\Delta \omega\|_{L^{q}}^{2} d \tau \leq C \int_{0}^{t}\|\nabla \times u\|_{L^{q}}^{2}+C\left(u_{0}, \omega_{0}, \theta_{0} ; T\right) .
$$

Employing (4.14) and (4.21) and then using the Sobolev embedding, we obtain

$$
\int_{0}^{t}\|\nabla \omega\|_{L^{\infty}} \leq C\left(u_{0}, \omega_{0}, \theta_{0} ; T\right) .
$$

Exactly as in the proof of (3.5), we also have

$$
\begin{aligned}
\ln \left(\|u\|_{H^{s}}^{2}+\|\omega\|_{H^{s}}^{2}+\|\theta\|_{H^{s}}^{2}\right) \leq & C \ln \left(\left\|u_{0}\right\|_{H^{s}}^{2}+\left\|\omega_{0}\right\|_{H^{s}}^{2}+\left\|\theta_{0}\right\|_{H^{s}}^{2}\right) \\
& +C \int_{0}^{t}\left(1+\|\nabla u\|_{L^{\infty}}+\|\nabla \omega\|_{L^{\infty}}+\|\nabla \theta\|_{L^{\infty}}\right) d \tau .
\end{aligned}
$$

Combining with (4.11), (4.15), (4.22) and (4.22), and then applying the Gronwall inequality, we conclude that

$$
\|u\|_{H^{s}}^{2}+\|\omega\|_{H^{s}}^{2}+\|\theta\|_{H^{s}}^{2} \leq C\left(u_{0}, \omega_{0}, \theta_{0} ; T\right) .
$$

The proof of Theorem 1.2 is completed.

\section{The Proof of Theorem 1.3}

In order to prove the global regularity part of Theorems 1.3] it suffices to get estimate (1.3) for all $T \in(0, \infty)$ for the smooth solutions of the system (1.2). Here, we divide it into the following two parts.

\subsection{Bound for $\|u\|_{L^{\infty}}$.}

Now, we recall the equation about the $\Omega=\nabla \times u$

$$
\Omega_{t}+u \cdot \nabla \Omega+2 \kappa \Delta \omega=\partial_{1} \theta .
$$

Taking scaler product (5.1) in $L^{2}$ by $|\Omega|^{p-2} \Omega(2<p<\infty)$, after integration by part, and using the incompressibility of $u$, we obtain the inequality

$$
\frac{1}{p} \frac{d}{d t}\|\Omega\|_{L^{p}}^{p} \leq\|\Delta \omega\|_{L^{p}}\|\Omega\|_{L^{p}}^{p-1}+\|\nabla \theta\|_{L^{p}}\|\Omega\|_{L^{p}}^{p-1} .
$$


Dividing by $\|\nabla \theta\|_{L^{p}}^{p-1}$, integrating in time and then using the Sobolev embedding yield

$$
\begin{aligned}
\|\Omega\|_{L^{p}} & \leq\left\|\Omega_{0}\right\|_{L^{p}}+\int_{0}^{t}\|\Delta \omega\|_{L^{p}} d \tau+\int_{0}^{t}\|\nabla \theta\|_{L^{p}} d \tau \\
& \leq\left\|\Omega_{0}\right\|_{L^{p}}+\int_{0}^{t}\|\Delta \omega\|_{L^{p}} d \tau+\int_{0}^{t}\left\|\nabla^{2} \theta\right\|_{L^{2}} d \tau \\
& \leq\left\|\Omega_{0}\right\|_{L^{p}}+C(T)\left(\int_{0}^{t}\|\Delta \omega\|_{L^{p}}^{2} d \tau\right)^{\frac{1}{2}}+\left(\int_{0}^{t}\left\|\nabla^{2} \theta\right\|_{L^{2}}^{2} d \tau\right)^{\frac{1}{2}},
\end{aligned}
$$

which implies that

$$
\|\Omega\|_{L^{p}}^{2} \leq\left\|\Omega_{0}\right\|_{L^{p}}^{2}+C(T)\left(\int_{0}^{t}\|\Delta \omega\|_{L^{p}}^{2} d \tau+\int_{0}^{t}\left\|\nabla^{2} \theta\right\|_{L^{2}}^{2} d \tau\right) .
$$

Using the Gronwall inequality and combining with (4.2) and (4.21), we finally get

$$
\|\Omega\|_{L^{p}} \leq C\left(u_{0}, \omega_{0}, \theta_{0} ; T\right) .
$$

Therefore, by the following Gagliardo-Nirenberg interpolation inequality in $\mathbb{R}^{2}$

$$
\|f\|_{L^{\infty}} \leq C\|f\|_{L^{2}}^{\frac{p-2}{2-2}}\|\nabla f\|_{L^{p}}^{\frac{p}{2 p-2}}, \quad f \in W^{1, p}, \quad p>2,
$$

and the Calderon-Zygmund inequality and (4.1), (5.3), we have

$$
\|u\|_{L^{\infty}} \leq C\|u\|_{L^{2}}^{\frac{p-2}{2 p-2}}\|\nabla u\|_{L^{p}}^{\frac{p}{2 p-2}} \leq C\|u\|_{L^{2}}^{\frac{p-2}{2 p-2}}\|\Omega\|_{L^{p}}^{\frac{p}{2 p-2}} \leq C\left(u_{0}, \omega_{0}, \theta_{0}, T, p\right) .
$$

\subsection{Bound for $\|\nabla \theta\|_{L^{\infty}}$.}

Taking scalar product (4.9) in $L^{2}$ by $\left|\nabla^{\perp} \theta\right|^{p-2} \nabla^{\perp} \theta(2<p<\infty)$ again, after integration by part, and using the incompressibility of $u$, the Hölder inequality, the Young inequality and the Calderon-Zygmund inequality, we get

$$
\begin{aligned}
& \frac{1}{p} \frac{d}{d t}\left\|\nabla^{\perp} \theta\right\|_{L^{p}}^{p}+\mu(p-1) \int\left|D^{2} \theta\right|^{2}\left|\nabla^{\perp} \theta\right|^{p-2} d x \\
& =\int \nabla^{\perp} \theta \cdot \nabla u\left|\nabla^{\perp} \theta\right|^{p-2} \nabla^{\perp} \theta d x+\int \nabla u\left|\nabla^{\perp} \theta\right|^{p-2} \nabla^{\perp} \theta d x \\
& \leq \int|u|^{2}\left|\nabla^{\perp} \theta\right|^{p} d x+\left.\frac{\mu(p-1)}{2} \int\left|\nabla^{2} \theta\right|^{2} \nabla^{\perp} \theta\right|^{p-2} d x+\|\nabla u\|_{L^{p}}\|\nabla \theta\|_{L^{p}}^{p-1} \\
& \leq C\|u\|_{L^{\infty}}^{2}\|\nabla \theta\|_{L^{p}}^{p}+\left.\frac{\mu(p-1)}{2} \int\left|\nabla^{2} \theta\right|^{2} \nabla^{\perp} \theta\right|^{p-2} d x+\|\Omega\|_{L^{p}}^{p}+\|\nabla \theta\|_{L^{p}}^{p},
\end{aligned}
$$

which implies that

$$
\frac{1}{p} \frac{d}{d t}\left\|\nabla^{\perp} \theta\right\|_{L^{p}}^{p}+\frac{\mu(p-1)}{2} \int\left|D^{2} \theta\right|^{2}\left|\nabla^{\perp} \theta\right|^{p-2} d x \leq C\left(1+\|u\|_{L^{\infty}}^{2}\right)\|\nabla \theta\|_{L^{p}}^{p}+\|\Omega\|_{L^{p}}^{p},
$$

which together with (5.3), (5.5) and the Gronwall inequality implies that

$$
\|\nabla \theta\|_{L^{p}} \leq C\left(u_{0}, \omega_{0}, \theta_{0}, T, p\right) .
$$

Taking operation $D^{2}$ on the third equation of (1.2), and then taking $L^{2}$ scalar product of this with $\left|D^{2} \theta\right|^{p-2} D^{2} \theta(2<p<\infty)$, after integration by part, and using the Hölder inequality, the Young inequality, Gagliardo-Nirenberg interpolation 
inequality (5.4) and the Calderon-Zygmund inequality, we deduce that

$$
\begin{aligned}
& \frac{1}{p} \frac{d}{d t}\left\|D^{2} \theta\right\|_{L^{p}}^{p}+\mu(p-1) \int\left|D^{3} \theta\right|^{2}\left|D^{2} \theta\right|^{p-2} d x \\
&=-\int D^{2}(u \cdot \nabla \theta)\left|D^{2} \theta\right|^{p-2} D^{2} \theta d x+\int D^{2} u\left|D^{2} \theta\right|^{p-2} D^{2} \theta d x \\
&=(p-1) \int D u \cdot \nabla \theta\left|D^{2} \theta\right|^{p-2} D^{3} \theta d x+(p-1) \int u \cdot D \nabla \theta\left|D^{2} \theta\right|^{p-2} D^{3} \theta d x \\
&+(p-1) \int D u\left|D^{2} \theta\right|^{p-2} D^{3} \theta d x \\
& \leq C\|\nabla \theta\|_{L^{\infty}}^{2}\|D u\|_{L^{p}}^{2}\left\|D^{2} \theta\right\|_{L^{p}}^{p-2}+\frac{\mu(p-1)}{2} \int\left|D^{3} \theta\right|^{2}\left|D^{2} \theta\right|^{p-2} d x+C\|u\|_{L^{\infty}}^{2}\left\|D^{2} \theta\right\|_{L^{p}}^{p} \\
&+\|D u\|_{L^{p}}^{2}\left\|D^{2} \theta\right\|_{L^{p}}^{p-2} \\
& \leq C\|\nabla \theta\|_{L^{p}}^{2-\frac{4}{p}}\|\Omega\|_{L^{p}}^{2}\left\|D^{2} \theta\right\|_{L^{p}}^{\frac{4}{p}+p-2}+\frac{\mu(p-1)}{2} \int\left|D^{3} \theta\right|^{2}\left|D^{2} \theta\right|^{p-2} d x+C\|u\|_{L^{\infty}}^{2}\left\|D^{2} \theta\right\|_{L^{p}}^{p} \\
&+\|\Omega\|_{L^{p}}^{2}\left\|D^{2} \theta\right\|_{L^{p}}^{p-2},
\end{aligned}
$$

where we have used the following interpolation inequality in $\mathbb{R}^{2}$

$$
\|f\|_{L^{\infty}} \leq C\|f\|_{L^{p}}^{1-\frac{2}{p}}\|\nabla f\|_{L^{p}}^{\frac{2}{p}}, \quad f \in W^{1, p}, \quad p>2 .
$$

Note $\frac{4}{p}+p-2<p$ when $p>2$, thanks to the estimates (5.3) (5.5) and (5.6), we obtain

$$
\frac{d}{d t}\left\|D^{2} \theta\right\|_{L^{p}}^{p} \leq C+C\left\|D^{2} \theta\right\|_{L^{p}}^{p}
$$

Using the Gronwall inequality yields that

$$
\left\|D^{2} \theta\right\|_{L^{p}} \leq C\left(u_{0}, \omega_{0}, \theta_{0}, T, p\right) .
$$

Furthermore, employing (5.9) and (5.8), and using Gagliardo-Nirenberg interpolation (5.7), we finally deduce that

$$
\|\nabla \theta\|_{L^{\infty}} \leq C\left(u_{0}, \omega_{0}, \theta_{0} ; T\right) \quad \forall t \in[0, T],
$$

where $C=C\left(\left\|u_{0}\right\|_{W^{2, p}},\left\|\omega_{0}\right\|_{W^{2, p}},\left\|\theta_{0}\right\|_{W^{2, p}}\right)$. Due to the embedding $H^{s}\left(\mathbb{R}^{2}\right) \hookrightarrow$ $W^{2, p}\left(\mathbb{R}^{2}\right)$, for all $s>2$ and $p>2$, and thus we attained estimate (1.3) for all $T \in(0, \infty)$ and for all $u_{0}, \omega_{0}, \theta_{0} \in H^{s}\left(\mathbb{R}^{2}\right)$ with $s>2$. The proof of Theorem 1.3 is completed.

acknowledgements: We are grateful to Professor Yi Zhou for his guidance and encouragement, which greatly improved our original manuscript.

\section{REFERENCES}

[1] Amann, H.: Maximal regularity for nonautonoumous evolution equations. Adv. Nonlinear Stud., 4, 417-430(2004)

[2] Aubin, J.: Un théorème de compacité. Comptes Rendus de l'Académie des Sciences, Paris., 256, 5042-5044 (1963)

[3] Bahouri, H., Chemin, J. Y., Danchin R.: Fourier analysis and nonlinear partial differential equations, Grundlehren der Mathematischen Wissenschaften, Springer-Verlag, Heidelberg, 2011

[4] Beale, J., Kato, T., Majda, A.: Remarks on the breakdown of smooth solutions for the 3-D Euler equations. Comm. Math. Phys., 94, 61-66(1984)

[5] Chemin, J. Y.: Perfect incompressible fluids, Oxford University Press, New York, 1998 
[6] Dong, B., Zhang, Z.: Global regularity of the 2D micropolar fluid flows with zero angular viscosity. J. Differential Equations., 249, 200-213(2010)

[7] Dong, B., Li, J., Wu, J.: Global well-posedness and large-time decay for the 2D micropolar equations. J. Differential Equations., 262, 3488-3523(2017)

[8] Eringen, A. C.: Theory of micropolar fluids. J. Math. Mech., 16, 1-18(1996)

[9] Foias, C., Manley, O., Temam, R.: Attractors for the Béenard problem: existence and physical bounds on their fractal dimension. Nonlinear Analysis (TMA), 11(8), 939-967(1987)

[10] Kato, T., Ponce, G.: Commutator estimates and the Euler and Navier-Stokes equations. Comm. Pure Appl. Math., 41(7), 891-907(1988)

[11] Kalita, P., Langa, J., Łukaszewicz, G.: Micropolar meets Newtonian. Rayleigh-Bénard problem, Physica D., 392, 57-80(2019)

[12] Popel, A., Regirer, S., Usick, P.: A continuum model of blood flow. Biorheology, 11, 427437(1974)

[13] Tarasinska, A.: Global attractor for heat convection problem in a micropolar fluid. Math. Meth. Appl. Sci., 29, 1215-1236(2006)

[14] Xue, L.: Well posedness and zero microrotation viscosity limit of the 2D micropolar fluid equations. Math. Methods Appl. Sci., 34, 1760-1777(2011)

[15] Xu, F., Chi, M.: Global regularity for the 2D micropolar Rayleigh-Bénard convection system with the zero diffusivity. Applied Mathematics Letters, 108, 106508(2020)

[16] Xu, F., Qiao, L., Zhang, M.: On the well-posedness for the 2D micropolar Rayleigh-Bénard convection problem. Z. Angew. Math. Phys., 72 17-29(2021)

Shanghai Center for Mathematical Sciences, Fudan University, Shanghai 200433, P.R. CHINA;

Email address: 19110840011@fudan.edu.cn 